\title{
Adolescent Atomoxetine Treatment in a Rodent Model of ADHD: Effects on Cocaine Self-Administration and Dopamine Transporters in Frontostriatal Regions
}

\author{
Sucharita S Somkuwar ${ }^{1,3}$, Chloe J Jordan ${ }^{2,3}$, Kathleen M Kantak ${ }^{2}$ and Linda P Dwoskin*,' \\ 'Department of Pharmaceutical Sciences, College of Pharmacy, University of Kentucky, Lexington, KY, USA; ' Department of Psychology, \\ Boston University, Boston, MA, USA
}

\begin{abstract}
Cocaine abuse and attention deficit/hyperactivity disorder (ADHD) are often comorbid. Preclinical research indicates that medial prefrontal (mPFC) and orbitofrontal (OFC) cortices are important neural substrates for both disorders. Using the spontaneously hypertensive rat $(\mathrm{SHR})$ model of $\mathrm{ADHD}$, we reported that adolescent treatment with the stimulant methylphenidate, a dopamine (DAT) and norepinephrine (NET) transporter inhibitor, enhanced cocaine self-administration during adulthood, and was associated with increased DAT function in mPFC. This study investigates the effects of atomoxetine ((R)- $N$-methyl- $\gamma$ - $(2$-methylphenoxy)benzenepropanamine hydrochloride) treatment, a selective NET inhibitor, during adolescence on cocaine self-administration and on DAT function and cell-surface expression in MPFC and OFC during adulthood. SHR acquired cocaine self-administration faster than Wistar-Kyoto and Wistar. Across cocaine doses, SHR earned more cocaine infusions and had higher progressive-ratio breakpoints than Wistar-Kyoto and Wistar, demonstrating that the SHR phenotype models comorbid ADHD and cocaine abuse. Prior atomoxetine treatment did not augment cocaine self-administration in SHR, but acquisition was enhanced in Wistar-Kyoto. No strain differences were found for DAT kinetic parameters or cellular localization in the vehicle controls. Atomoxetine did not alter DAT kinetic parameters or localization in SHR mPFC. Rather, atomoxetine decreased $V_{\max }$ and DAT cell surface expression in SHR OFC, indicating that inhibition of NET by atomoxetine treatment during adolescence indirectly reduced DAT function and trafficking to the cell surface in OFC, specifically in the ADHD model. Thus, atomoxetine, unlike methylphenidate, does not enhance vulnerability to cocaine abuse in SHR and may represent an important alternative for teens with ADHD when drug addiction is a concern.

Neuropsychopharmacology (20I3) 38, 2588-2597; doi: I0.I038/npp.20 I3. I63; published online 24 July 2013
\end{abstract}

Keywords: addiction; attention deficit/hyperactivity disorder; cocaine; dopamine transporter; atomoxetine; spontaneously hypertensive rat

\section{INTRODUCTION}

Attention deficit/hyperactivity disorder (ADHD) affects $8-12 \%$ of children, and up to $5 \%$ of adults, making it one of the most prevalent disorders (Biederman et al, 2010). Adults with ADHD have a higher risk of developing substance-use disorders compared with individuals without ADHD (Wilens et al, 1998). In particular, individuals with ADHD have a $35 \%$ higher incidence of cocaine abuse compared with the general population (Levin et al, 1999), and children with ADHD are two times as likely to use cocaine during adulthood (Lee et al, 2011).

*Correspondence: Dr LP Dwoskin, Department of Pharmaceutical Sciences, College of Pharmacy, University of Kentucky, 789 South Limestone, 465 Biological Pharmaceutical Complex, Lexington, KY 40536-0596, USA, Tel: +I 859257 4743, Fax: + I 8592577585, E-mail: Idwoskin@email.uky.edu

${ }^{3}$ These authors contributed equally to this work

Received 25 February 2013; revised 12 June 2013; accepted I July 2013; accepted article preview online 3 July 2013
One explanation for comorbid ADHD and cocaine abuse may be commonalities in neuronal substrates. Hypoactivation of the orbitofrontal cortex (OFC) and dorsolateral prefrontal cortex (DLPFC) is evident in both disorders and leads to deficits in working memory, decision making, and response inhibition (Adinoff et al, 2003; Bolla et al, 2003; Burgess et al, 2010; Cubillo et al, 2011; Wilcox et al, 2011). With respect to rodents, behavioral flexibility, working memory, and sustained attention are regulated by both the medial prefrontal cortex (mPFC) and OFC (Floresco et al, 2009). Moreover, mPFC and OFC also regulate cocaineseeking behavior in outbred rats self-administering cocaine (Di Pietro et al, 2006; Kantak et al, 2013). As the mPFC in rodents is functionally analogous to DLPFC in primates (Uylings et al, 2003), these findings suggest that mPFC and OFC function is critical for understanding the mechanisms underlying comorbid ADHD and cocaine abuse.

There is a paucity of information concerning the effects of ADHD medications on the comorbidity of ADHD and cocaine abuse. Primarily, two categories of medications are used to treat ADHD: stimulants (eg, methylphenidate) and 
non-stimulants (eg, atomoxetine $((R)-N$-methyl- $\gamma$-(2-methylphenoxy)-benzenepropanamine hydrochloride)). Methylphenidate is a dopamine and norepinephrine transporter (DAT and NET, respectively) inhibitor (Richelson and Pfenning, 1984), whereas atomoxetine is a selective NET inhibitor (Bolden-Watson and Richelson, 1993). Although methylphenidate is the first-line treatment for $\mathrm{ADHD}$, atomoxetine has efficacy nearly comparable to methylphenidate in reducing ADHD symptoms (Garnock-Jones and Keating, 2009).

Clinical reports suggest that methylphenidate treatment initiated in childhood may be protective against cocaine addiction (Wilens et al, 2003), or alternatively, not modify cocaine abuse liability in ADHD individuals (Molina et al, 2013). However, a positive correlation was reported between age of initiation of methylphenidate treatment and cocaine abuse during adulthood (Mannuzza et al, 2008), such that lifetime rates of cocaine abuse were higher when treatment was initiated in early adolescence. Preclinical models using carefully controlled experimental conditions may be valuable for evaluating mechanisms underlying these controversial clinical results. Previous work using spontanously hypertensive rats (SHR), a well-established model of ADHD (Kantak et al, 2008; Sagvolden et al, 2005), found that methylphenidate treatment during adolescence increased cocaine self-administration in adult SHR compared with untreated SHR and compared with methylphenidate-treated Wistar-Kyoto (WKY) or Wistar (WIS) control rats (Harvey et al, 2011). In the latter report, repeated administration of methylphenidate during adolescence increased the selfadministration efficacy for a range of doses of cocaine, without altering sensitivity for cocaine in SHR. Increased vulnerability to cocaine self-administration in SHR may be due to methylphenidate-induced increases in DAT function in $\mathrm{mPFC}$ relative to untreated SHR and methylphenidatetreated WKY or WIS (Somkuwar et al, 2013). These findings from the SHR model are complimentary with observations in humans, such that previously medicated ADHD individuals have higher striatal DAT density compared with nonADHD individuals (Fusar-Poli et al, 2012). Striatal DAT function is also elevated in cocaine users (Mash et al, 2002). However, cortical DAT expression in ADHD and in cocaine users has not been reported.

Atomoxetine, unlike methylphenidate, has very low affinity for DAT (Heal et al, 2008). However, selective inhibition of NET by atomoxetine increases extracellular concentrations of both norepinephrine and dopamine in PFC (Arnsten, 2009), because NET is responsible primarily for dopamine clearance in this brain region (Moron et al, 2002). In outbred rats, atomoxetine reduces cocaine-seeking and cocaine cue-induced reinstatement (Economidou et al, 2011; Janak et al, 2012). However, effects of atomoxetine on cocaine self-administration and on DAT function and cell surface expression in SHR have not been determined. This study tests the hypothesis that, in contrast to the effects of methylphenidate treatment, treatment with a pharmacologically relevant dose of atomoxetine (Bymaster et al, 2002) during adolescence does not increase vulnerability for cocaine self-administration in adult SHR after the atomoxetine treatment has been discontinued. To identify longterm changes in dopaminergic systems, DAT function and expression in $\mathrm{MPFC}, \mathrm{OFC}$, and striatum in adult SHRs were evaluated following the administration of a pharmacologically relevant dose of atomoxetine during adolescence. Further, to identify changes in DAT function that may influence cocaine self-administration, neurochemical studies were conducted in rats at the same age at which cocaine self-administration was initiated.

\section{MATERIALS AND METHODS}

\section{Subjects}

Male WIS/Cr, WKY/Cr and SHR/Cr rats (Charles River Laboratories, Wilmington, MA, Kingston, NY or Raleigh, NC) arrived on postnatal day 25 (P25). SHR served as an animal model of ADHD, and WKY and WIS as inbred and outbred comparator strains, respectively. Experiments 1, 2, and 3 used the same group of rats. Experiment 4 used a separate group of rats. Experiment 5 again used a separate group of rats. Housing has been described previously (Harvey et al, 2011) and in the Supplementary Materials. From P28 through P55, rats received atomoxetine or vehicle (see below) Monday through Friday to mimic the weekend 'medication holiday' often recommended for individuals with ADHD (American Academy of Pediatrics Committee on Children With Disabilities and Committee on Drugs, 1996). For rats used in the behavioral studies, an intravenous catheter was implanted on P67 (see Supplementary Materials for details). Protocols were approved by the Institutional Animal Care and Use Committee at Boston University and at the University of Kentucky, and were performed in accordance with the 1996 version of the NIH Guide for the Care and Use of Laboratory Animals.

\section{Drugs}

Atomoxetine (R)-N-methyl-g-(2-methylphenoxy)-benzenepropanamine hydrochloride (Tocris Biosciences, Ellisville, MO; $0.3 \mathrm{mg} / \mathrm{ml}$ ) was dissolved in $0.9 \%$ sterile physiological saline and injected intraperitoneally at a dose of $0.3 \mathrm{mg} / \mathrm{kg}$. This relatively low dose was chosen to increase extracellular norepinephrine concentrations in PFC, although this dose also has been shown to increase extracellular dopamine concentrations in PFC through inhibition of NET (Bymaster et al, 2002). An intraperitoneal route was utilized because of poor oral bioavailability of atomoxetine in rats (Mattiuz et al, 2003). For intravenous self-administration studies, cocaine hydrochloride (NIDA, Bethesda, MD) was dissolved in sterile $0.9 \%$ physiological saline containing $3 \mathrm{IU}$ of heparin per $\mathrm{ml}$. A cocaine unit dose of $0.3 \mathrm{mg} / \mathrm{kg}$ was used for training, and doses from 0.003 to $1.0 \mathrm{mg} / \mathrm{kg}$ were used to evaluate dose-response functions.

Experiment 1: Acquisition of cocaine self-administration (fixed ratio). Experiment 1 determined the speed at which WIS, WKY, and SHR acquired cocaine self-administration, and evaluated strain-dependent effects of adolescent atomoxetine treatment on acquisition. Sessions $(2 \mathrm{~h})$ were conducted daily Monday-Friday beginning on P77 in lighted chambers described in Supplementary Materials. Rats were allowed to press the active lever (left or right, counterbalanced across rats) for a $0.3 \mathrm{mg} / \mathrm{kg}$ cocaine infusion under a fixed ratio 1 (FR1) schedule of reinforcement. Responses on the inactive lever were recorded, but 
had no consequences. Animals received no external inducements to respond on either lever (spontaneous acquisition). A stimulus light located above the active lever was illuminated upon receipt of a cocaine infusion and remained illuminated during a 20-s timeout period, during which additional infusions could not be earned, but lever responses were counted. The house light was extinguished during the timeout. Acquisition of cocaine self-administration was defined as earning $\geqslant 20$ infusions in a 2 -h session for two consecutive sessions, and discriminating the active from inactive lever by a factor of 2 or greater (Harvey et al, 2011).

Experiment 2: Cocaine dose-response functions (FR). Experiment 2 evaluated the efficacy of cocaine reinforcement in WIS, WKY, and SHR, and identified strain-dependent effects of adolescent atomoxetine treatment on responding maintained by a range of cocaine doses under an FR1 schedule. Following achievement of acquisition criterion, rats continued under an FR1 schedule of $0.3 \mathrm{mg} / \mathrm{kg}$ cocaine delivery until active lever responses and infusions varied $<10 \%$ across five consecutive sessions. A range of cocaine unit doses (0.003, 0.01. 0.03, 0.1, and $1.0 \mathrm{mg} / \mathrm{kg}$ per infusion) was then substituted in random order two times each week (Tuesdays and Fridays). The $0.3 \mathrm{mg} / \mathrm{kg}$ training dose was available on intervening days. Following determination of FR1 cocaine dose-response functions, baseline responding was re-established for the $0.3 \mathrm{mg} / \mathrm{kg}$ dose for 2-3 days before beginning Experiment 3.

Experiment 3: Cocaine dose-response functions (progressive ratio). Experiment 3 assessed the motivating influence of cocaine reinforcement in WIS, WKY, and SHR, and identified strain-dependent effects of atomoxetine treatment during adolescence on progressive-ratio (PR) breakpoints across a range of cocaine unit doses. The PR schedule of Loh and Roberts (1990) was implemented, such that response requirements on the active lever increased exponentially for each subsequent cocaine infusion. Self-administration sessions terminated when rats failed to meet the response requirement within $1 \mathrm{~h}$. The last FR completed was defined as the PR breakpoint. Baseline responding under the PR was established for the $0.3 \mathrm{mg} / \mathrm{kg}$ dose for five consecutive sessions, after which test doses $(1.0,0.1$, and $0.01 \mathrm{mg} / \mathrm{kg}$ per infusion) were substituted in descending order.

Experiment 4: Dopamine uptake assay. Experiment 4 assessed DAT function in $\mathrm{MPFC}, \mathrm{OFC}$, and striatum of WIS, WKY, and SHR treated with atomoxetine or vehicle. Kinetic analysis of $\left[{ }^{3} \mathrm{H}\right]$ dopamine uptake into $\mathrm{mPFC}, \mathrm{OFC}$, and striatal synaptosomes was conducted using a previously published procedure (Marusich et al, 2011) with minor modifications. Purified synaptosomal suspensions from $\mathrm{mPFC}, \mathrm{OFC}$, and striatum from one atomoxetine- and one vehicle-treated rat of the same strain and age (P77-P84) were prepared as detailed in Supplementary Materials. Briefly, mPFC, OFC, and striatal samples were incubated for $5 \mathrm{~min}$ in the absence or presence of an excess concentration of nomifensine, a DAT inhibitor, to determine nonspecific $\left[{ }^{3} \mathrm{H}\right]$ dopamine uptake, and paroxetine and desipramine to prevent $\left[{ }^{3} \mathrm{H}\right]$ dopamine uptake by serotonin and norepinephrine transporters, respectively. Subsequently, 1 of 7 final concentrations $(0.01-1.0 \mu \mathrm{M})$ of $\left[{ }^{3} \mathrm{H}\right]$ dopamine was added to the assay buffer and incubations continued for $\mathrm{mPFC}$ (5 $\mathrm{min})$, OFC (5 min), and striatal (10 min) synaptosomal suspensions. Specific $\left[{ }^{3} \mathrm{H}\right]$ dopamine uptake was obtained by subtracting nonspecific uptake from total uptake; these values were used to determine kinetic parameters $\left(V_{\max }\right.$ and $\left.K_{\mathrm{m}}\right)$ using the commercially available GraphPad Prism 5.0 program (GraphPad Software, San Diego, CA).

Experiment 5: DAT cellular distribution assay. Experiment 5 assessed DAT cellular distribution in $\mathrm{mPFC}$, OFC, and striatum of WIS, WKY, and SHR treated with atomoxetine or vehicle. Synaptosomal pellets of mPFC, $\mathrm{OFC}$, and striatum were resuspended in $1.25 \mathrm{ml}$ (mPFC and $\mathrm{OFC}$ ) or in $3 \mathrm{ml}$ (striatum) of ice-cold sucrose solution. Biotinylation and western blotting assays were performed using a previously published method (Somkuwar et al, 2013) detailed in Supplementary Materials. Briefly, synaptosomal suspensions were incubated with sulfo-NHS biotin to label all surface proteins. Synaptosomes were lysed by sonication and incubation in Triton X-100 buffer. Total protein fractions were obtained by centrifugation. Twothirds of the total protein fractions were incubated with avidin beads to separate non-biotinylated (supernatant) from avidin-conjugated biotinylated fractions (pellet). Total, non-biotinylated, and biotinylated fractions were subjected to gel electrophoresis and western blotting, and subsequently probed for DAT protein, $\mathrm{Na}^{+} / \mathrm{K}^{+}$ATPase (plasma-membrane-enriched protein), and PP2A (intracellular protein) for determining efficiency of biotinylation, and $\beta$-actin (cytoskeletal protein, loading control) to ascertain protein loading. Band density, expressed as relative optical density, was determined for DAT and $\beta$-actin using the ImageJ software (http://imagej.nih.gov/ij).

\section{Data Analyses}

Dependent measures for self-administration experiments included sessions to reach acquisition criterion (square root transformed before analysis), cocaine infusions earned, active, and inactive lever responses, and PR breakpoints. Dependent measures for DAT functional assays included $K_{\mathrm{m}}$ (log transformed before analysis) and $V_{\max }(\mathrm{pmol} / \mathrm{mg} / \mathrm{min}$; atomoxetine-treated rats were normalized as percent of vehicle-treated control of the same strain). For the cellular distribution assay, DAT from each fraction (total, intracellular, and surface) for each brain region was normalized to $\beta$-actin levels in the same sample. Dependent measures were analyzed by one-factor (strain), two-factor (strain $\times$ treatment, drug dose $\times$ treatment, or drug dose $\times$ strain), or three-factor (drug dose $\times$ strain $\times$ treatment) ANOVAs, with repeated measures for dose. Post hoc Tukey's tests were used in behavioral studies and Tukey's or one-sample $t$-tests (compared with a hypothetical value of 100) for matched subjects in neurochemical studies. Outliers in neurochemical studies were removed before analysis using the Grubbs test (GraphPad Software; http://www.graphpad.com/quickcalcs/Grubbs1.cfm). 


\section{RESULTS}

\section{Experiment 1: Acquisition of Cocaine Self-} Administration (FR)

Sessions to reach the acquisition criterion for the $0.3 \mathrm{mg} / \mathrm{kg}$ dose is shown in Figure 1. Strains differed significantly $(\mathrm{F}(2$, $47)=7.0, p \leqslant 0.002$ ), and there was a trend for a strain $\times$ treatment interaction $(\mathrm{F}(2,47)=2.7, p \leqslant 0.07)$. Overall, SHR acquired cocaine self-administration faster than WKY and WIS ( $p \leqslant 0.04$ and 0.002 , respectively). Treatment comparisons within each strain revealed that in WKY, acquisition of cocaine self-administration was faster after atomoxetine than vehicle $(p \leqslant 0.03)$. Atomoxetine did not alter acquisition speed in SHR or WIS. Strain comparisons within each treatment revealed that in vehicle-treated rats, SHR acquired cocaine self-administration faster than WKY $(p \leqslant 0.02)$. In atomoxetine-treated rats, both SHR and WKY acquired cocaine self-administration faster than WIS $(p \leqslant 0.01$ and 0.05 , respectively).

Analyses of active and inactive responses as well as infusions earned at criterion also were performed (Supplementary Table S1 and Supplementary Figure S1). These analyses confirm strain-level differences, with SHR emitting more active lever responses and earning more cocaine infusions than WKY or WIS, overall. These analyses also revealed that at criterion, atomoxetine treatment increased active lever responses exclusively in SHR. Moreover, numbers of inactive lever responses emitted were not different between strains and between treatment conditions, and rats discriminated the active from inactive lever by a factor of 2 or greater (Supplementary Table S1).

\section{Experiment 2: Cocaine Dose-Response Functions (FR)}

Cocaine dose-response functions based on the number of infusions earned under FR1 are shown in Figure 2a. For the

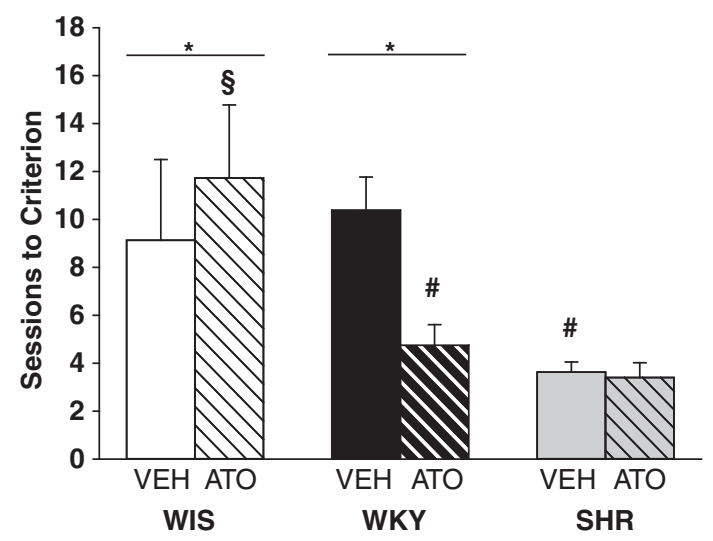

Figure I Number of sessions to reach criterion for acquisition of cocaine self-administration. Experiments were performed using adult Wistar (WIS; white bars), Wistar-Kyoto (WKY; black bars), and spontaneously hyperactive (SHR; gray bars) rats after discontinuation of adolescent treatment with atomoxetine (ATO; striped bars) or vehicle (VEH; solid bars). Values are mean \pm SEM $(n=8-11$ per strain and treatment). ${ }^{*} p \leqslant 0.05$ compared with SHR (denoted by horizontal line under the symbol). ${ }^{\#} p \leqslant 0.05$ compared with VEH-treated WKY. ${ }^{\S} p \leqslant 0.05$ compared with ATO-treated WKY and SHR. three-way ANOVA, strain $(\mathrm{F}(2,43)=18.4, p \leqslant 0.001)$ and dose $(F(5,215)=185.5, p \leqslant 0.001)$ differed, and there was a strain $\times$ dose interaction $(\mathrm{F}(10,215)=9.3, p \leqslant 0.001)$. The treatment factor and its interactions with strain and/or dose were not significant. Overall, SHR earned more cocaine infusions than WKY and WIS ( $p \leqslant 0.001)$. Further testing of the strain $\times$ dose interaction indicated that SHR earned more infusions than WKY for cocaine doses ranging from 0.003 to $0.3 \mathrm{mg} / \mathrm{kg}(p \leqslant 0.04)$, and more infusions than WIS for cocaine doses ranging from 0.003 to $0.1 \mathrm{mg} / \mathrm{kg}(p \leqslant 0.01$ except at $0.01 \mathrm{mg} / \mathrm{kg}$, where $p \leqslant 0.08)$. In addition, WIS earned more infusions than WKY at $0.1 \mathrm{mg} / \mathrm{kg}(p \leqslant 0.001)$. No strain differences were observed at $1.0 \mathrm{mg} / \mathrm{kg}$. Analyses of the cocaine dose-response functions based on the number of active lever responses were similar to the above number of infusions earned (Supplementary Figure S2).

\section{Experiment 3: Cocaine Dose-Response Functions (PR)}

Cocaine dose-response functions based on the last FR completed under the PR schedule are shown in Figure $2 \mathrm{~b}$. For the three-way ANOVA, strain $(\mathrm{F}(2,42)=10.1, p \leqslant 0.001)$ and dose $(\mathrm{F}(3,126)=53.3, p \leqslant 0.001)$ differed. The treatment factor and its interactions with strain and/or dose were not significant. Overall, SHR had higher breakpoints than WKY and WIS $(p \leqslant 0.001$ and 0.01 , respectively). Further analysis of the dose factor revealed that animals maintained the highest breakpoints at $1.0 \mathrm{mg} / \mathrm{kg}$, which differed from all other doses, and maintained the lowest breakpoints at $0.01 \mathrm{mg} / \mathrm{kg}$, which also differed from all other doses $(p \leqslant 0.001-0.03)$. Breakpoints maintained by 0.3 and $0.1 \mathrm{mg} / \mathrm{kg}$ did not differ from each other. PR breakpoints based on infusions earned and active lever responses also were analyzed and results were similar to the last FR completed measure (Supplementary Figures S3 and S4).

\section{Experiment 4: Dopamine Uptake}

$K_{\mathrm{m}}$ values for $\left[{ }^{3} \mathrm{H}\right]$ dopamine uptake in $\mathrm{mPFC}, \mathrm{OFC}$, and striatum did not differ among strain or treatment groups (Supplementary Table S2). Also, the $V_{\max }$ for $\left[{ }^{3} \mathrm{H}\right]$ dopamine uptake by DAT in MPFC, OFC, and striatum did not differ between strains treated with vehicle (Figures $3 a, 4 a$ and $5 a$ ). Atomoxetine treatment did not alter $V_{\max }$ in mPFC in any strain when compared with the corresponding vehicle control. Also, there were no differences in $V_{\max }$ in $\mathrm{mPFC}$ between strains treated with atomoxetine (Figure $3 \mathrm{a}$ ). Conversely, with respect to the OFC, atomoxetine decreased $V_{\max }$ for $\left[{ }^{3} \mathrm{H}\right]$ dopamine uptake in SHR $(t(7)=2.42, p \leqslant 0.05)$ and WIS $(t(5)=5.67, p \leqslant 0.005)$ by $25 \%$ and $51 \%$ of vehicle control, respectively (Figure 4a). Furthermore, there were strain differences in OFC in the atomoxetine-treated groups. Specifically, $V_{\max }$ was lower for atomoxetine-treated SHR and WIS (14\% and 55\%, respectively) compared with atomoxetine-treated $\mathrm{WKY}(\mathrm{F}(2,21)=8.22, p \leqslant 0.005)$. Atomoxetine also decreased $V_{\max }$ in SHR striatum $(t(6)=2.74$, $p \leqslant 0.05$ ) by $18 \%$ of vehicle control (Figure $5 \mathrm{a}$ ). However, $V_{\max }$ in the striatum did not differ among the atomoxetinetreated groups. 

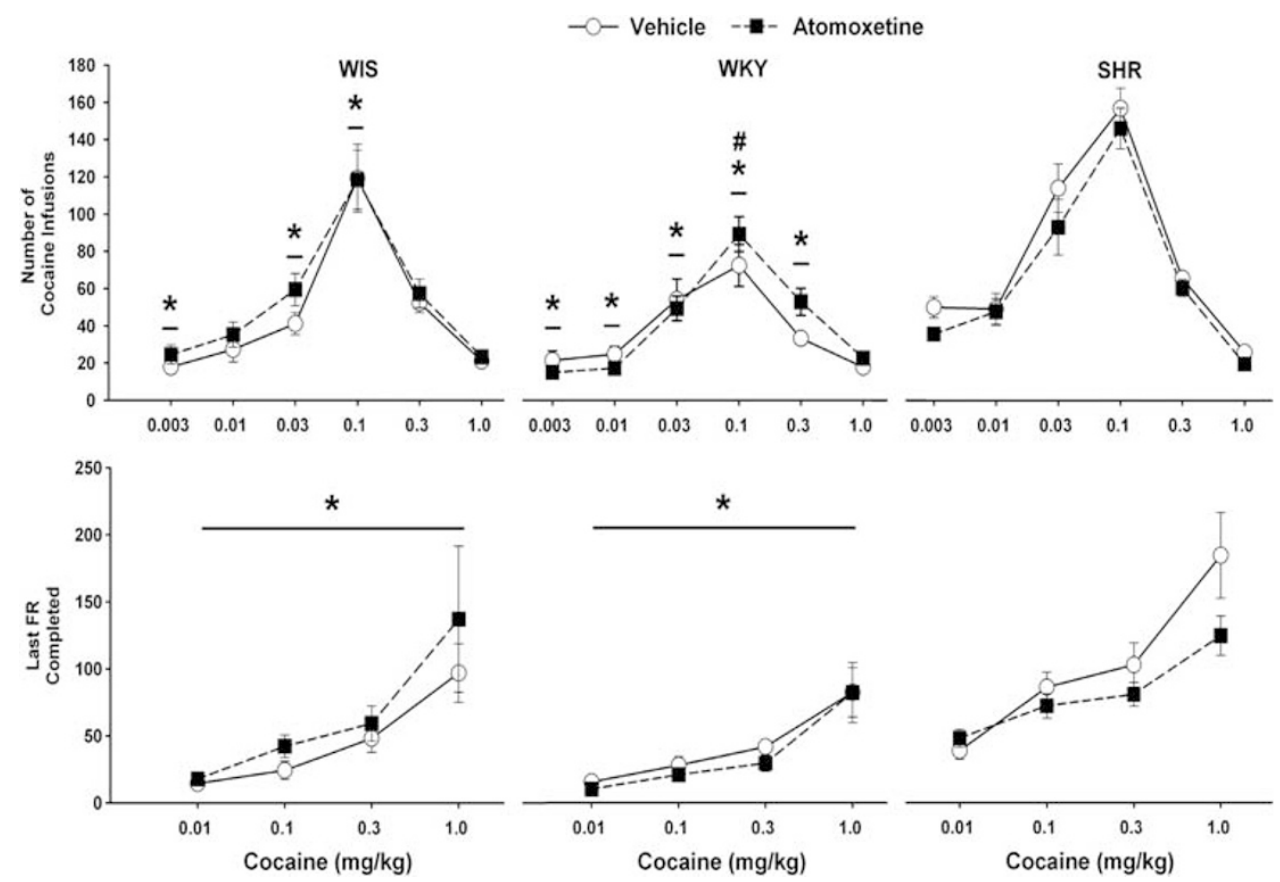

Figure 2 (a) Cocaine dose-response functions based on the number of infusions earned under a fixed ratio I (FRI) schedule $(n=8-9$ per strain and treatment). (b) Progressive ratio breakpoints based on the last FR completed ( $n=8$ per strain and treatment). Experiments were performed using adult Wistar (WIS), Wistar-Kyoto (WKY), and spontaneously hypertensive (SHR) rats following discontinuation of adolescent treatment with atomoxetine (ATO; squares, dashed lines) or vehicle (VEH; circles, solid lines). Values are mean \pm SEM $* p \leqslant 0.05$ compared with SHR overall (denoted by horizontal line under the symbol). ${ }^{\#} p \leqslant 0.05$ compared with the same dose in WIS.
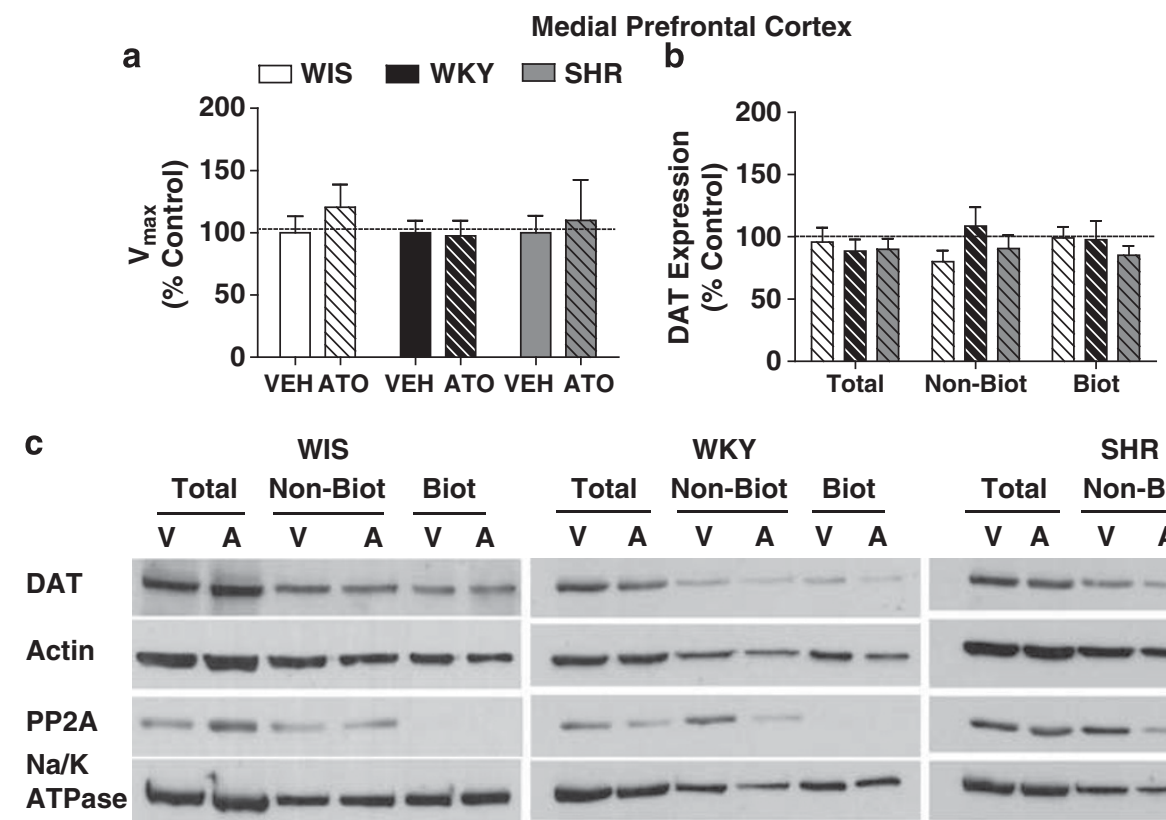

Figure 3 Dopamine transporter (DAT) $V_{\max }$ and cell surface distribution in the medial prefrontal cortex (mPFC) of adult Wistar (WIS), Wistar-Kyoto (WKY) rats, and spontaneously hypertensive rats (SHRs) following discontinuation of adolescent treatment with atomoxetine (ATO or vehicle (VEH). (a) $V_{\max }$ values are mean $\pm \mathrm{SEM}$ pmol/mg/min expressed as a percent of $\mathrm{VEH}$ control ( $n=6-10$ per strain and treatment). $V_{\text {max }}$ values for WIS, WKY, and SHR VEH control groups were $3.0 \pm 0.4,2.4 \pm 0.3$, and $3.9 \pm 0.7 \mathrm{pmol} / \mathrm{mg} / \mathrm{min}$, respectively, and were not different from each other. (b) DAT expression values are mean \pm SEM arbitrary units for DAT density following ATO treatment expressed as a percentage of VEH control (dotted line, see Supplementary Materials for VEH control values). (c) Representative blots for DAT cellular distribution between total, non-biotinylated (Non-Biot; intracellular), and biotinylated (Biot; cell surface) fractions in mPFC synaptosomes from ATO-treated (a) and VEH-treated (V) WIS, WKY, and SHRs. Actin was used to normalize DAT expression for each individual sample, whereas $\mathrm{Na}^{+} / \mathrm{K}^{+}$ATPase and PP2A served to ascertain efficiency of biotinyation of the surface proteins $(n=6-7$ per strain and treatment). 


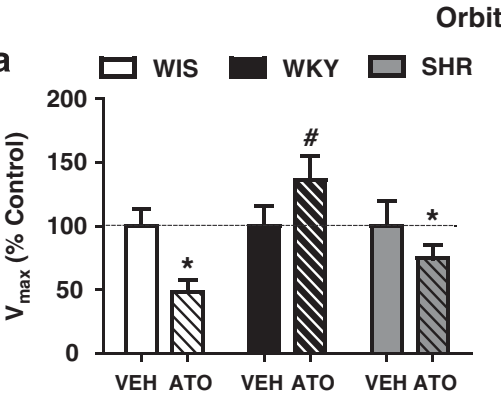

Orbitofrontal Cortex

C
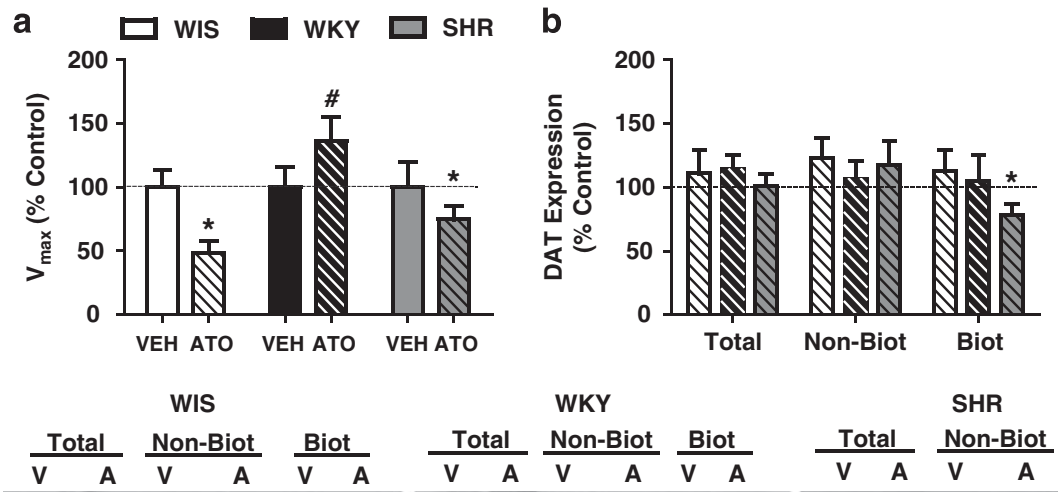

WKY

DAT

V A V A V A
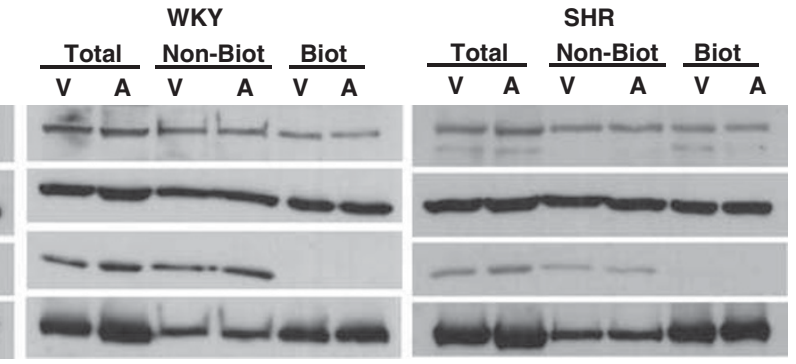

Figure 4 Dopamine transporter (DAT) $V_{\max }$ and cell surface distribution in the orbitofrontal cortex (OFC) of adult Wistar (WIS), Wistar-Kyoto (WKY) rats, and spontaneously hypertensive rats (SHRs) following discontinuation of adolescent treatment with atomoxetine (ATO or vehicle (VEH). (a) DAT $V_{\text {max }}$ values are mean $\pm \mathrm{SEM}$ pmol/mg/min expressed as a percent of VEH control ( $n=6-10$ per strain and treatment). $V_{\text {max }}$ Values for WIS, WKY, and SHR VEH control groups were $3.5 \pm 0.4,2.8 \pm 0.5$, and $4.3 \pm 0.9 \mathrm{pmol} / \mathrm{mg} / \mathrm{min}$, respectively, and were not different from each other. (b) DAT expression values are mean \pm SEM arbitrary units for DAT density following ATO treatment expressed as a percentage of VEH control (dotted line, see Supplementary Materials for VEH control values). (c) Representative blots for distribution of DAT between total, non-biotinylated (Non-Biot; intracellular), and biotinylated (Biot; cell surface) fractions in OFC synaptosomes from ATO-treated (a) and VEH -treated (V) WIS, WKY, and SHRs. Actin was used to normalize DAT expression for each individual sample, whereas $\mathrm{Na}^{+} / \mathrm{K}^{+}$ATPase and protein phosphatase A (PP2A) served to ascertain the efficiency of biotinyation of surface proteins ( $n=6-7$ per strain and treatment). *P $\leqslant 0.05$ compared with the VEH control value of I 00\%; ${ }^{*} \leqslant 0.05$ compared with ATO-treated WIS and SHR.

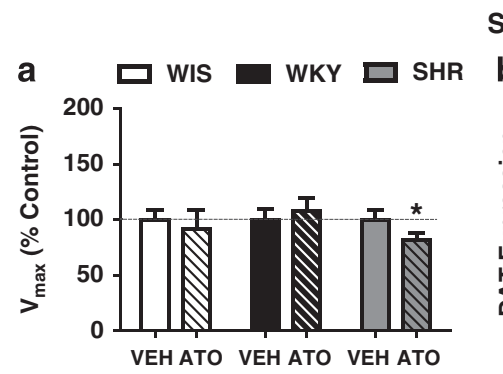

Striatum

\section{b}

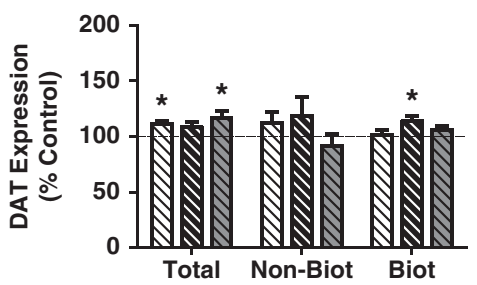

C

WIS

WKY

SHR
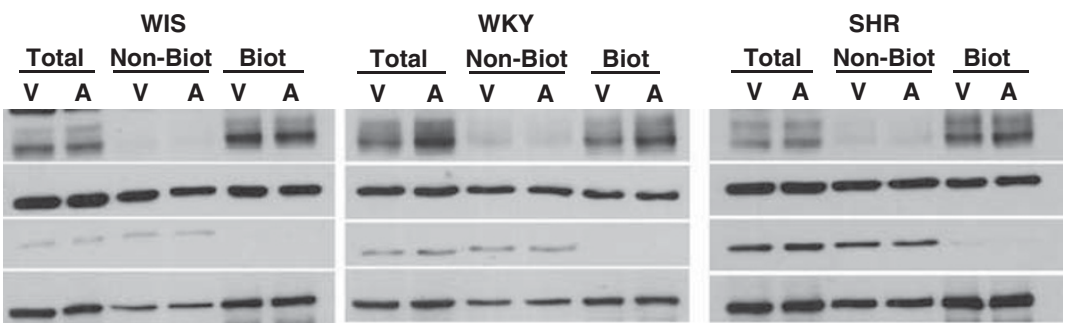

Figure 5 Dopamine transporter (DAT) $V_{\max }$ and cell surface distribution in the striatum of adult Wistar (WIS), Wistar-Kyoto (WKY) rats, and spontaneously hypertensive rats (SHRs) following discontinuation of adolescent treatment with atomoxetine (ATO or vehicle (VEH). (a) DAT $V_{\text {max }}$ values are mean $\pm \mathrm{SEM}$ pmol/mg/min expressed as a percent of VEH control $\left(n=5-6\right.$ per strain and treatment). $V_{\max }$ values for WIS, WKY, and SHR VEH control groups were $19.0 \pm 1.7,15.4 \pm 2.3$, and $17.3 \pm 1.8 \mathrm{pmol} / \mathrm{mg} / \mathrm{min}$, respectively, and were not different from each other. (b) DAT expression values are mean \pm SEM arbitrary units for DAT density following ATO treatment expressed as a percentage of VEH control (dotted line, see Supplementary Materials for VEH control values). (c) Representative blots for distribution of DAT between total, non-biotinylated (Non-Biot; intracellular), and biotinylated (Biot; cell surface) fractions in striatal synaptosomes from ATO-treated (a) and VEH-treated (V) WIS, WKY, and SHRs. Actin was used to normalize DAT expression for each individual sample, whereas $\mathrm{Na}^{+} / \mathrm{K}^{+}$ATPase and protein phosphatase A (PP2A) served to ascertain the efficiency of biotinyation of surface proteins ( $n=5-6$ per strain and treatment). *P $\leqslant 0.05$ compared with the VEH control value of $100 \%$. 


\section{Experiment 5: DAT Cellular Distribution Assay}

In the vehicle-treated groups, no between-strain differences in DAT cellular distribution were found in $\mathrm{MPFC}, \mathrm{OFC}$, and striatum (Supplementary Table S3). Atomoxetine treatment did not alter DAT cellular distribution in MPFC (Figures 3b and c). However in OFC, atomoxetine treatment significantly decreased (22\% of vehicle control) DAT expression in the surface fraction only in SHR (Figures $4 \mathrm{~b}$ and $\mathrm{c}$; $t(7)=2.50, p \leqslant 0.05)$. DAT cellular distribution in OFC in the atomoxetine-treated groups was not different between the strains (Figures $4 \mathrm{~b}$ and $\mathrm{c}$ ). In the striatum, atomoxetine treatment during adolescence increased total DAT expression in SHR ( $18 \%$ of vehicle control; $t(7)=2.76, p \leqslant 0.05$ ) and WIS ( $12 \%$ of vehicle control; $t(6)=4.35, p \leqslant 0.05$; Figures $5 \mathrm{~b}$ and $\mathrm{c})$. Also, surface DAT in the striatum of WKY was increased $(13 \%$ of vehicle control; $t(7)=2.88$, $p \leqslant 0.05$; Figures $5 \mathrm{~b}$ and $\mathrm{c}$ ). DAT cellular distribution in the striatum did not differ among atomoxetine-treated groups (Figures $5 \mathrm{~b}$ and $\mathrm{c}$ ).

\section{DISCUSSION}

\section{Strain Differences in Behavior and DAT Neurochemistry}

Compared with inbred WKY and outbred WIS comparator strains, SHR acquired cocaine self-administration faster, and showed greater intake and higher breakpoints across a range of cocaine unit doses under FR1 and PR schedules. These results suggest that SHR exhibit a vulnerable cocaine self-administration phenotype, characterized by faster acquisition, higher efficacy, and a greater motivating influence of cocaine reinforcement. Vulnerability is reflected by vertical shifts in FR and PR dose-response functions (Piazza et al, 2000). Thus, these findings further verify the utility of the SHR for modeling comorbid cocaine abuse and ADHD (Harvey et al, 2011).

Comparator strains did not differ, except that WIS had greater cocaine intake and made more active lever responses than WKY for $0.1 \mathrm{mg} / \mathrm{kg}$ cocaine under FR1. This dose produced peak rates of responding in all strains. Cocaine doses commonly abused in people and those associated with peak rates of responding in rhesus monkeys produce similar levels of striatal DAT occupancy (Wilcox et al, 2002). This suggests that strain differences in cocaine self-administration may reflect strain differences in DAT function or expression. Compared with WIS, WKY have decreased DAT density in nucleus accumbens (Jiao et al, 2003). In this study, however, there were no strain differences in DAT function in $\mathrm{MPFC}, \mathrm{OFC}$, or striatum under vehicle conditions. Further, no differences were found in DAT cellular distribution in MPFC, OFC, and striatum among SHR, WKY, and WIS, which is in agreement with some previous findings (Jiao et al, 2003; Li et al, 2007), but not others (Pandolfo et al, 2012; Roessner et al, 2010). Inconsistencies in striatal DAT expression may be explained by the use of $\left[{ }^{3} \mathrm{H}\right] \mathrm{GBR} 12935$ to assess expression, as this radioligand binds to both intracellular and cell surface DAT protein (Roessner et al, 2010). Further, prior history of the subjects, including participation in behavioral assays, may explain some inconsistencies between studies in striatal DAT function and expression
(Pandolfo et al, 2012). With respect to striatal cell surface DAT expression, there was a trend suggesting that WKY had lower DAT expression than SHR and WIS, although this was not associated with differences in striatal DAT function (Harvey et al, 2011; Womersley et al, 2011). Thus, based in the literature, cocaine may be a more efficacious reinforcer in SHR and WIS than WKY, because of greater DAT cell surface expression within the reward circuit in SHR and WIS.

\section{Effects of Atomoxetine Treatment}

Following discontinuation of methylphenidate treatment in adolescent SHR, an increase in cocaine self-administration was observed; and importantly, this was not observed in the control WKY and WIS rats (Harvey et al, 2011). These findings appear to differ from those of other investigators reporting no increase in cocaine self-administration after discontinuing methylphenidate treatment (Gill et al, 2012; Thanos et al, 2007); however, rats or monkeys that did not have an ADHD phenotype were used in these studies. Thus, our results with the control strains (not expressing the ADHD phenotype) are in agreement with the latter findings. Further, our results using SHR, which display the ADHD phenotype, extend the literature, and moreover, are in agreement with clinical reports that teens with ADHD treated with stimulant medication have greater liability for cocaine abuse (Harvey et al, 2011; Mannuzza et al, 2008). Interestingly, when the ADHD stimulant treatment was initiated during childhood, a decreased drug abuse liability was found in adulthood (Wilens et al, 2003). Taken together, this approach using an animal model of ADHD is appropriate and clinically relevant for determining the effects of alternate ADHD medications such as atomoxetine.

Adolescent atomoxetine treatment did not alter cocaine self-administration behavior in SHR, with one exception. Atomoxetine-treated SHR made more active lever responses at the acquisition criterion under FR1, without having greater cocaine intake. This indicates that SHR made more responses during the $20 \mathrm{~s}$ timeout/cue light presentation period following each cocaine infusion. Acute atomoxetine pretreatment has cognitive enhancing effects via increased noradrenergic transmission (Gamo et al, 2010; Janak and Corbit, 2011). Thus, it is possible that chronic atomoxetine treatment increased the salience of cocaine-paired cues in SHR during acquisition of cocaine self-administration via inhibition of norepinephrine uptake. As chronic atomoxetine treatment during adolescence selectively increases NET mRNA in the OFC during adulthood (Sun et al, 2012), the OFC may be an important site of action for atomoxetine-induced changes in cocaine cue salience.

The results of this report suggest that alterations in DAT function and expression in OFC after atomoxetine treatment may also be of importance to cocaine self-administration behavior. Adolescent atomoxetine treatment increased the speed of acquisition of cocaine self-administration in adult WKY, but not SHR and WIS, and correspondingly decreased DAT function in OFC of SHR and WIS, but not WKY. In our previous work, atomoxetine treatment during adolescence was shown to differentially influence performance on a strategy set shifting task as well (Harvey et al, 2013). Specifically, learning speed during the initial discrimination phase was decreased in SHR and 
increased in WKY and WIS after atomoxetine. During the set shift phase, atomoxetine improved learning accuracy exclusively in SHR, suggesting strain-dependent effects of atomoxetine on prefrontal cortical functions (Floresco et al, 2009). In addition to decreases in $V_{\max }$ of dopamine uptake at DAT in OFC, atomoxetine decreased cell surface expression of DAT in OFC in SHR, revealing an underlying trafficking-dependent mechanism for the observed decrease in OFC DAT function. It has been shown that repeated application of dopamine decreases DAT function and expression in the striatum in vivo and in a heterologous expression system in vitro (Gulley et al, 2002). As dopamine is cleared by NET in MPFC and OFC, chronic atomoxetine treatment will increase extracellular dopamine concentrations in these brain regions, which may lead to decreased DAT surface expression, and subsequently to decreased DAT function as observed in the SHR OFC. In WIS, the identical atomoxetine treatment decreased DAT function in OFC, but did not decrease DAT cell surface expression, indicating a trafficking-independent mechanism. Taken together, these results suggest that atomoxetine may be protective against further enhancement of vulnerability to cocaine self-administration in SHR, via a long-lived reduction in DAT function and/or surface expression in OFC. Another variable that may confer this protection in SHR is the failure of adolescent atomoxetine treatment in SHR to increase DAT function in MPFC during adulthood. In our previous studies, adolescent methylphenidate treatment increased $\mathrm{mPFC}$ DAT function in SHR (Somkuwar et al, 2013) and further enhanced vulnerability to cocaine self-administration in SHR (Harvey et al, 2011) during adulthood.

Atomoxetine unexpectedly decreased DAT function and increased total DAT expression in SHR striatum. The increase in total striatal DAT expression may be a compensatory response to the decrease in DAT function. Also, atomoxetine increased total DAT expression in WIS and surface expression in WKY. Thus, atomoxetine alterations in striatal DAT expression were not specific to the strain with the ADHD phenotype. These results may reveal neuronal cross-talk between dopaminergic neurons in the striatum and noradrenergic neurons in cortex, where atomoxetine has a direct effect at NET (Swanson et al, 2006).

\section{CONCLUSIONS}

This study demonstrates that the SHR phenotype models comorbid cocaine abuse and ADHD. Contrary to previous findings with methylphenidate, atomoxetine treatment did not further enhance vulnerability to cocaine self-administration in SHR. Although additional work is needed to confirm mechanisms and signaling pathways involved, our work suggests that the protection by atomoxetine against further enhanced cocaine abuse vulnerability in SHR may occur through decreased DAT function and decreased DAT cell surface localization in OFC, and sustained DAT function in $\mathrm{mPFC}$. Taken together, the behavioral and neurochemical results suggest that atomoxetine may be a suitable alternative to stimulant treatment in ADHD teens in whom the risk of drug abuse may be a concern.
Moreover, while these studies do not raise critical concerns about the safety of atomoxetine, they do emphasize the importance of accurate diagnosis of ADHD. In the WKY control, atomoxetine speeded acquisition of cocaine selfadministration and did not alter OFC DAT. Misdiagnosis of $\mathrm{ADHD}$, and subsequent atomoxetine treatment in teens could result in a more rapid development of abuse of cocaine.

\section{FUNDING AND DISCLOSURE}

This study was supported by Grant R01 DA011716 and a Kentucky Opportunity Fellowship (SS). The authors declare no conflict of interest.

\section{ACKNOWLEDGEMENTS}

We thank Britahny Baskin, Marcela Cruz, Angelica DellaMorte, Malak El Quessny, and Agripina Deaciuc for technical assistance. We thank Britahny Baskin, Marcela Cruz, Angelica DellaMorte, Malak El Quessny, and Agripina Deaciuc for technical assistance.

\section{Author contributions}

SS and CJ contributed equally to the manuscript and are cofirst authors. KK and LD were responsible for the study concept and design. SS and CJ collected data, analyzed results, and drafted the manuscript. KK and LD provided important intellectual content. All authors critically reviewed and edited the content and approved the final version for publication.

\section{REFERENCES}

Adinoff B, Devous MD Sr, Cooper DB, Best SE, Chandler P, Harris T et al (2003). Resting regional cerebral blood flow and gambling task performance in cocaine-dependent subjects and healthy comparison subjects. Am J Psychiatry 160: 1892-1894.

American Academy of Pediatrics Committee on Children With Disabilities and Committee on Drugs (1996). Medication for children with attentional disorders. Pediatrics 98(Part 1): 301-304.

Arnsten AF (2009). Toward a new understanding of attentiondeficit hyperactivity disorder pathophysiology: an important role for prefrontal cortex dysfunction. CNS Drugs 23(Suppl 1): 33-41.

Biederman J, Petty CR, Evans M, Small J, Faraone SV (2010). How persistent is ADHD? A controlled 10-year follow-up study of boys with ADHD. Psychiatry Res 177: 299-304.

Bolden-Watson C, Richelson E (1993). Blockade by newlydeveloped antidepressants of biogenic amine uptake into rat brain synaptosomes. Life Sci 52: 1023-1029.

Bolla KI, Eldreth DA, London ED, Kiehl KA, Mouratidis M, Contoreggi C et al (2003). Orbitofrontal cortex dysfunction in abstinent cocaine abusers performing a decision-making task. NeuroImage 19: 1085-1094.

Burgess GC, Depue BE, Ruzic L, Willcutt EG, Du YP, Banich MT (2010). Attentional control activation relates to working memory in attention-deficit/hyperactivity disorder. Biol Psychiatry 67: 632-640.

Bymaster FP, Katner JS, Nelson DL, Hemrick-Luecke SK, Threlkeld PG, Heiligenstein JH et al (2002). Atomoxetine increases extracellular levels of norepinephrine and dopamine 
in prefrontal cortex of rat: a potential mechanism for efficacy in attention deficit/hyperactivity disorder. Neuropsychopharmacology 27: 699-711.

Cubillo A, Halari R, Giampietro V, Taylor E, Rubia K (2011). Fronto-striatal underactivation during interference inhibition and attention allocation in grown up children with attention deficit/hyperactivity disorder and persistent symptoms. Psychiatry Res 193: 17-27.

Di Pietro NC, Black YD, Kantak KM (2006). Context-dependent prefrontal cortex regulation of cocaine self-administration and reinstatement behaviors in rats. Eur J Neurosci 24: 3285-3298.

Economidou D, Dalley JW, Everitt BJ (2011). Selective norepinephrine reuptake inhibition by atomoxetine prevents cueinduced heroin and cocaine seeking. Biol Psychiatry 69: 266-274.

Floresco SB, Zhang Y, Enomoto T (2009). Neural circuits subserving behavioral flexibility and their relevance to schizophrenia. Behav Brain Res 204: 396-409.

Fusar-Poli P, Rubia K, Rossi G, Sartori G, Balottin U (2012). Striatal dopamine transporter alterations in ADHD: pathophysiology or adaptation to psychostimulants? A meta-analysis. Am J Psychiatry 169: 264-272.

Gamo NJ, Wang M, Arnsten AF (2010). Methylphenidate and atomoxetine enhance prefrontal function through alpha2adrenergic and dopamine D1 receptors. J Am Acad Child Adolesc Psychiatry 49: 1011-1023.

Garnock-Jones KP, Keating GM (2009). Atomoxetine: a review of its use in attention-deficit hyperactivity disorder in children and adolescents. Paediatr Drugs 11: 203-226.

Gill KE, Pierre PJ, Daunais J, Bennett AJ, Martelle S, Gage HD et al (2012). Chronic treatment with extended release methylphenidate does not alter dopamine systems or increase vulnerability for cocaine self-administration: a study in nonhuman primates. Neuropsychopharmacology 37: 2555-2565.

Gulley JM, Doolen S, Zahniser NR (2002). Brief, repeated exposure to substrates down-regulates dopamine transporter function in Xenopus oocytes in vitro and rat dorsal striatum in vivo. J Neurochem 83: 400-411.

Harvey RC, Jordan CJ, Tassin DH, Moody KR, Dwoskin LP, Kantak KM (2013). Performance on a strategy set shifting task during adolescence in a genetic model of attention deficit/hyperactivity disorder: methylphenidate vs. atomoxetine treatments. Behav Brain Res 244: 38-47.

Harvey RC, Sen S, Deaciuc A, Dwoskin LP, Kantak KM (2011). Methylphenidate treatment in adolescent rats with an attention deficit/hyperactivity disorder phenotype: cocaine addiction vulnerability and dopamine transporter function. Neuropsychopharmacology 36: 837-847.

Heal DJ, Smith SL, Kulkarni RS, Rowley HL (2008). New perspectives from microdialysis studies in freely-moving, spontaneously hypertensive rats on the pharmacology of drugs for the treatment of ADHD. Pharmacol Biochem Behav 90: 184-197.

Janak PH, Bowers MS, Corbit LH (2012). Compound stimulus presentation and the norepinephrine reuptake inhibitor atomoxetine enhance long-term extinction of cocaine-seeking behavior. Neuropsychopharmacology 37: 975-985.

Janak PH, Corbit LH (2011). Deepened extinction following compound stimulus presentation: noradrenergic modulation. Learn Mem 18: 1-10.

Jiao X, Pare WP, Tejani-Butt S (2003). Strain differences in the distribution of dopamine transporter sites in rat brain. Progr Neuro-Psychopharmacol Biol Psychiatry 27: 913-919.

Kantak KM, Singh T, Kerstetter KA, Dembro KA, Mutebi MM, Harvey RC et al (2008). Advancing the spontaneous hypertensive rat model of attention deficit/hyperactivity disorder. Behav Neurosci 122: 340-357.

Kantak KM, Yager LM, Brisotti MF (2013). Impact of medial orbital cortex and medial subthalamic nucleus inactivation, individually and together, on the maintenance of cocaine self-administration behavior in rats. Behav Brain Res 238: 1-9.

Lee SS, Humphreys KL, Flory K, Liu R, Glass K (2011). Prospective association of childhood attention-deficit/hyperactivity disorder (ADHD) and substance use and abuse/dependence: a metaanalytic review. Clin Psychol Rev 31: 328-341.

Levin FR, Evans SM, Kleber HD (1999). Practical guidelines for the treatment of substance abusers with adult attention-deficit hyperactivity disorder. Psychiatric Serv 50: 1001-1003.

Li Q, Lu G, Antonio GE, Mak YT, Rudd JA, Fan M et al (2007). The usefulness of the spontaneously hypertensive rat to model attention-deficit/hyperactivity disorder (ADHD) may be explained by the differential expression of dopamine-related genes in the brain. Neurochem Int 50: 848-857.

Loh EA, Roberts DC (1990). Break-points on a progressive ratio schedule reinforced by intravenous cocaine increase following depletion of forebrain serotonin. Psychopharmacology 101: 262-266.

Mannuzza S, Klein RG, Truong NL, Moulton JL 3rd, Roizen ER, Howell KH et al (2008). Age of methylphenidate treatment initiation in children with ADHD and later substance abuse: prospective follow-up into adulthood. Am J Psychiatry 165: 604-609.

Marusich JA, Darna M, Charnigo RJ, Dwoskin LP, Bardo MT (2011). A multivariate assessment of individual differences in sensation seeking and impulsivity as predictors of amphetamine self-administration and prefrontal dopamine function in rats. Exp Clin Psychopharmacol 19: 275-284.

Mash DC, Pablo J, Ouyang Q, Hearn WL, Izenwasser S (2002). Dopamine transport function is elevated in cocaine users. J Neurochem 81: 292-300.

Mattiuz EL, Ponsler GD, Barbuch RJ, Wood PG, Mullen JH, Shugert RL et al (2003). Disposition and metabolic fate of atomoxetine hydrochloride: pharmacokinetics, metabolism, and excretion in the Fischer 344 rat and beagle dog. Drug Metab Dispos 31: 88-97.

Molina BS, Hinshaw SP, Eugene Arnold L, Swanson JM, Pelham WE, Hechtman L et al (2013). Adolescent substance use in the multimodal treatment study of attention-deficit/hyperactivity disorder (ADHD) (MTA) as a function of childhood ADHD, random assignment to childhood treatments, and subsequent medication. J Am Acad Child Adolesc Psychiatry 52: 250-263.

Moron JA, Brockington A, Wise RA, Rocha BA, Hope BT (2002). Dopamine uptake through the norepinephrine transporter in brain regions with low levels of the dopamine transporter: evidence from knock-out mouse lines. J Neurosci 22(2): 389-395.

Pandolfo P, Machado NJ, Kofalvi A, Takahashi RN, Cunha RA (2012). Caffeine regulates frontocorticostriatal dopamine transporter density and improves attention and cognitive deficits in an animal model of attention deficit hyperactivity disorder. Eur Neuropsychopharmacol 23: 317-28.

Piazza PV, Deroche-Gamonent V, Rouge-Pont F, Le Moal M (2000). Vertical shifts in self-administration dose-response functions predict a drug-vulnerable phenotype predisposed to addiction. J Neurosci 20: 4226-4232.

Richelson E, Pfenning M (1984). Blockade by antidepressants and related compounds of biogenic amine uptake into rat brain synaptosomes: most antidepressants selectively block norepinephrine uptake. Eur J Pharmacol 104: 277-286.

Roessner V, Sagvolden T, Dasbanerjee T, Middleton FA, Faraone SV, Walaas SI et al (2010). Methylphenidate normalizes elevated dopamine transporter densities in an animal model of the attention-deficit/hyperactivity disorder combined type, but not to the same extent in one of the attention-deficit/hyperactivity disorder inattentive type. Neuroscience 167: 1183-1191.

Sagvolden T, Russell VA, Aase H, Johansen EB, Farshbaf M (2005). Rodent models of attention-deficit/hyperactivity disorder. Biol Psychiatry 57: 1239-1247. 
Somkuwar SS, Darna M, Kantak KM, Dwoskin LP (2013). Adolescence methylphenidate treatment in a rodent model of attention deficit/hyperactivity disorder: dopamine transporter function and cellular distribution in adulthood. Biochem Pharmacol 86: 309-316.

Sun H, Cocker PJ, Zeeb FD, Winstanley CA (2012). Chronic atomoxetine treatment during adolescence decreases impulsive choice, but not impulsive action, in adult rats and alters markers of synaptic plasticity in the orbitofrontal cortex. Psychopharmacology (Berl) 219: 285-301.

Swanson CJ, Perry KW, Koch-Krueger S, Katner J, Svensson KA, Bymaster FP (2006). Effect of the attention deficit/hyperactivity disorder drug atomoxetine on extracellular concentrations of norepinephrine and dopamine in several brain regions of the rat. Neuropharmacology 50: 755-760.

Thanos PK, Michaelides M, Benveniste H, Wang GJ, Volkow ND (2007). Effects of chronic oral methylphenidate on cocaine selfadministration and striatal dopamine D2 receptors in rodents. Pharmacol Biochem Behav 87: 426-433.

Uylings HB, Groenewegen HJ, Kolb B (2003). Do rats have a prefrontal cortex? Behav Brain Res 146: 3-17.
Wilcox CE, Teshiba TM, Merideth F, Ling J, Mayer AR (2011). Enhanced cue reactivity and fronto-striatal functional connectivity in cocaine use disorders. Drug Alcohol Depend 115: 137-144.

Wilcox KM, Lindsey KP, Votaw JR, Goodman MM, Martarello L, Carroll FI et al (2002). Self-administration of cocaine and the cocaine analog RTI-113: relationship to dopamine transporter occupancy determined by PET neuroimaging in rhesus monkeys. Synapse 43: 78-85.

Wilens TE, Biederman J, Mick E (1998). Does ADHD affect the course of substance abuse? Findings from a sample of adults with and without ADHD. Am J Addict/Am Acad Psychiatrists Alcohol Addict 7: 156-163.

Wilens TE, Faraone SV, Biederman J, Gunawardene S (2003). Does stimulant therapy of attention-deficit/hyperactivity disorder beget later substance abuse? A meta-analytic review of the literature. Pediatrics 111: 179-185.

Womersley JS, Hsieh JH, Kellaway LA, Gerhardt GA, Russell VA (2011). Maternal separation affects dopamine transporter function in the spontaneously hypertensive rat: an in vivo electrochemical study. Behav Brain Funct 7: 49.

Supplementary Information accompanies the paper on the Neuropsychopharmacology website (http://www.nature.com/npp) 